\title{
TrustShadow: Secure Execution of Unmodified Applications with ARM TrustZone
}

\author{
Le Guan*, Peng Liu*, Xinyu Xing*, Xinyang Ge*, \\ Shengzhi Zhang ${ }^{\dagger}$, Meng $\mathrm{Yu}^{\ddagger}$, Trent Jaeger* \\ *Pennsylvania State University \\ \{lug14, pliu, xxing\}@ist.psu.edu, \{tjaeger\}@cse.psu.edu \\ \#Microsoft Research

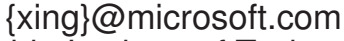 \\ ${ }^{\dagger}$ Florida Institute of Technology \\ \{zhangs\}@cs.fit.edu \\ ¥University of Texas at San Antonio \\ \{meng.yu\}@utsa.edu
}

\begin{abstract}
The rapid evolution of Internet-of-Things (IoT) technologies has led to an emerging need to make them smarter. A variety of applications now run simultaneously on an ARMbased processor. For example, devices on the edge of the Internet are provided with higher horsepower to be entrusted with storing, processing and analyzing data collected from IoT devices. This significantly improves efficiency and reduces the amount of data that needs to be transported to the cloud for data processing, analysis and storage. However, commodity OSes are prone to compromise. Once they are exploited, attackers can access the data on these devices. Since the data stored and processed on the devices can be sensitive, left untackled, this is particularly disconcerting.

In this paper, we propose a new system, TrustShadow that shields legacy applications from untrusted OSes. TrustShadow takes advantage of ARM TrustZone technology and partitions resources into the secure and normal worlds. In the secure world, TrustShadow constructs a trusted execution environment for security-critical applications. This trusted environment is maintained by a lightweight runtime system that coordinates the communication between applications and the ordinary OS running in the normal world. The runtime system does not provide system services itself. Rather, it forwards requests for system services to the ordinary OS, and verifies the correctness of the responses. To demonstrate the efficiency of this design, we prototyped TrustShadow on a real chip board with ARM TrustZone support, and evaluated its performance using both microbenchmarks and real-world applications. We showed TrustShadow introduces only negligible overhead to real-world applications.
\end{abstract}

Permission to make digital or hard copies of all or part of this work for personal or classroom use is granted without fee provided that copies are not made or distributed for profit or commercial advantage and that copies bear this notice and the full citation on the first page. Copyrights for components of this work owned by others than ACM must be honored. Abstracting with credit is permitted. To copy otherwise, or republish, to post on servers or to redistribute to lists, requires prior specific permission and/or a fee. Request permissions from permissions@acm.org.

MobiSys '17, June 19-23, 2017, Niagara Falls, NY, USA.

(c) 2017 ACM. ISBN 978-1-4503-4928-4/17/06 . \$ $\$ 15.00$

DOI: http://dx.doi.org/10.1145/3081333.3081349

\section{Keywords}

Malicious Operating Systems; ARM TrustZone; IoT; Trusted Execution

\section{INTRODUCTION}

The emerging Internet of Things (IoT) technologies have enabled more and more isolated "things" to collect, process, analyze, and exchange data. To become smart, IoT devices tend to be built atop ARM-based multi-programming platforms, in which multiple programs run simultaneously on commodity Operating Systems (OSes). This allows them to install product-ready applications and saves effort and budget in application development.

For example, edge computing $[9,8]$ is a decentralized computing infrastructure that connects IoT devices to process their data on other, more powerful devices that are on - or close to - the network edge. Examples of such edge devices include industrial controllers, smart gateway and routers, embedded servers, and even automotive in-vehicle infotainment etc. Compared to a central data center in the cloud, edge devices are geographically closer to the IoT devices. Thus, they can respond to IoT devices more quickly, making them more suitable to environments where operations are time-critical or Internet connectivity is poor. In telemedicine scenarios, edge devices could run sophisticated analytics and turn around results in real time [15]. As another example, a smart 3D-printer [19] can directly download an STL (STereoLithography) file from the "MakerBot Digital Store" [19], convert it into GCODE by running a slicer program, and feed the GCODE to the actuator.

Unfortunately, the security provided by commodity OSes in the multi-programming platforms is often inadequate. Once an OS is compromised, attackers gain complete access to the data on a system. Since these devices may often deal with confidential data, possibly subject to laws and regulations, this is particularly disconcerting.

To address this problem, a straightforward reaction is to safeguard applications against the OSes potentially vulnerable to exploitable bugs or misconfiguration. Prior efforts on this explore executing applications that handle sensitive data in separate virtual machines (e.g., [13, 26, 43]), taking advantage of hardware features (e.g., [6, 34, 33]) or retrofitting commodity OSes (e.g. [16]). Unfortunately, 
these solutions are not applicable to the aforementioned IoT multi-programming platforms.

First, these devices do not have the hardware features typically available on PCs. To be energy efficient, these devices generally incorporate ARM Cortex-A processors, making the techniques that rely on unique hardware completely futile (e.g., Haven [6] based on Intel SGX). Second, these devices do not have abundant computational resources in comparison with PCs or a data center in the cloud. Thus, it is not realistic to adopt to these devices those techniques specifically designed for PCs or data centers. Last but not least, some techniques previously proposed require radical changes to applications and OSes, which poses a substantial barrier to their adoption. This is especially true in the scenario where device manufacturers would like to retain compatibility with existing applications.

In this paper, we address the aforementioned issues by developing TrustShadow. TrustShadow is a system that shields legacy applications from a compromised OS. By taking advantage of ARM TrustZone technology [4], our system constructs a trusted execution environment for securitycritical applications. Different from some existing techniques, TrustShadow does not radically change existing OSes. Rather, it utilizes a lightweight runtime system to coordinate communications between applications and untrusted OSes. As such, TrustShadow requires no changes to existing applications either.

More specifically, we develop TrustShadow with a runtime system running in the TrustZone of an ARM processor. The runtime manages the page tables for applications locally in an isolated secure environment, and ensures their virtual memory cannot be accessed by an untrusted OS running outside the environment. To accommodate the execution of applications in a lightweight manner, the runtime does not incorporate complicated system services. Rather, it forwards application requests for system services to the untrusted OS, similar to Proxos [43]. To guarantee security, the runtime verifies return values from system services to defeat Iago attacks [11], and interposes context switches between the applications and the untrusted OS. Considering an application might interact with file I/O, the runtime system also encrypts the data before revealing it to the untrusted OS for storing.

With the design above, TrustShadow protects legacy applications from the untrusted OSes running them. As a result, developers no longer need to re-engineer applications in order to run them on IoT devices. Since TrustShadow does not implement system services itself, the complexity of Trusted Computing Base (TCB) is reduced, making TrustShadow less vulnerable to exploits. To the best of our knowledge, TrustShadow is the first solution on ARM-based IoT devices that allows an unmodified application to run protected from attacks from untrusted OSes

In summary, this paper makes the following contributions.

- We propose a system - TrustShadow- for ARM-based multi-programming platforms. It can protect securitycritical applications from untrusted OSes without the requirement of re-engineering the applications.

- We introduce a runtime system within TrustShadow. It accommodates the execution of Linux applications with a lightweight forwarding-and-verifying mechanism.
- We implemented TrustShadow on a real chip (SoC) board with the ARM TrustZone support with only about $5.3 \mathrm{~K}$ lines of code (LOC) in the secure world, and $300 \mathrm{LOC}$ in the normal world. Using microbenchmarks and real world software, we showed that TrustShadow imposes only negligible performance overhead.

The rest of the paper is organized as follows. Section 2 and 3 present related work and the background of TrustZone, respectively. Section 4 discusses our threat model. Section 5 describes the overview of TrustShadow. Section 6 and 7 introduce our design and prototype implementation in detail. We present the evaluation of TrustShadow in Section 8 , followed by some discussion in Section 9. Finally, we conclude the paper in Section 10.

\section{RELATED WORK}

As is described in Section 1, prior research primarily focuses on taking advantage of virtual machines, hardware features and radical code re-engineering to protect applications from compromised OSes. In this section, we summarize these works and describe why they are not suitable for IoT devices with more details.

Hypervisors and Virtual Machines. To protect an application from a compromised OS, one research effort focuses on utilizing hypervisor to construct trusted execution environment for applications. Systems following this design principle include Overshadow [13], CHAOS [12], SP ${ }^{3}$ [46], Inktag [26], etc. They encrypt address space for an application under protection through a hypervisor, so that a compromised OS can only view the address space of the application in ciphertext. Using the hypervisor, they also verify the integrity of memory contents, and thus ensure a compromised OS cannot jeopardize the execution of the application. Similar to these techniques, another research effort focuses on escalating protection with virtual machines. For example, Terra [21] and Proxos [43] allocate a dedicated VM for an application, making it resistant to a malicious OS.

While these systems have been shown to be effective in shielding applications, they are an overkill for resourceconstrained IoT devices, and sometimes cannot be adopted by IoT devices. First, deploying a hypervisor-based system cannot provide the best (native) performance for the already performance-hungry IoT devices [20]. Second, virtualization extension used in InkTag etc., is a new hardware feature for the ARM platform, and is missing for many existing ARM devices ${ }^{1}$. Third, ARM has recently released the new IoT-oriented Cortex-M processor series which incorporate TrustZone extension, not virtualization extension [3]. This meets our speculation that virtualization is not suitable for resource-constrained IoT devices.

From the security perspective, hypervisor or virtual machine based solutions relies on hypervisor, which is already struggling with its own security problems due to increasing TCB size [18, 17]. In this work, TrustShadow harnesses TrustZone technology to mediate communication between OS and applications, which eliminates complex, error-prone resource allocation in a hypervisor.

Hardware Features. Research in the past also explores using various hardware features to protect applications from

\footnotetext{
${ }^{1}$ ARM released virtualization extension in the year 2010 [2].
} 
untrusted OSes. For example, Haven [6] takes advantage of Intel Software Guard eXtension (SGX) [35] to safeguard applications. More specifically, it harnesses SGX to instantiate a secure region of address space, and then protects execution of applications within that region from malicious privilege code. In addition to Intel SGX, Trusted Platform Module (TPM) is also used for shielding applications from a potentially malicious OS. For example, both Flicker [34] and TrustVisor [33] utilize TPM to isolate the execution of sensitive code. As is described in Section 1, IoT devices generally incorporate ARM Cortex-A processors which do not have the aforementioned hardware features. As a result, previous techniques based on those cannot be applicable.

Trusted Language Runtime (TLR) [39], VeriUI [32] and Trust0TP [42] utilize ARM TrustZone technology for shielding applications. TLR implement a small runtime capable of interpreting .NET managed code inside the secure world. By splitting mobile application into secure part and nonsecure part, the secure part of the app is never exposed to the untrusted OSes. VeriUI utilizes TrustZone to provide a trustworthy setting for handling passwords. TrustOTP harnesses TrustZone to protect the confidentiality of the OneTime-Password against a malicious mobile OS. While these works take advantage of TrustZone, they require modifications to applications in order to be under protected. This poses a substantial barrier to their adoptions.

Code Instrumentation. Virtual Ghost [16] is another research endeavor on protecting applications from a hostile OS. Different from those techniques discussed above, it uses compiler techniques and run-time checking to implement a mechanism similar to InkTag within the OS kernel. Since the compiler instrumentation and run-time checking introduce more privilege code to kernel, not only does it increase TCB of a computer system but also imposes performance overhead, making it not suitable to energy-efficient, computation-lightweight IoT devices.

\section{TRUSTZONE}

In this section, we present the background of ARM TrustZone technology. To be more specific, we briefly describe its architecture, address space controller and memory management unit (MMU).

\subsection{Architecture}

ARM TrustZone partitions all of the System-on-Chip (SoC) hardware and software resources in one of two worlds - the secure world for the security subsystem, and the normal world for everything else. With this partition, a single physical processor core can safely and efficiently execute code from both the normal world and the secure world in a time-sliced fashion. When the processor executes code in the normal world, it enters a non-secure state in which the processor can only access resources in the normal world. Otherwise, it is in a secure state in which the processor can access resources resided in both worlds.

To determine whether program execution is in the secure or normal world, ARM TrustZone extends a Non-Secure bit (NS-bit) on the AMBA Advanced eXtensble Interface (AXI) bus. With this NS-bit, the processor can check permissions on the access. To manage switches to and from the secure world, TrustZone provides monitor mode software which ensures the state of the world that the processor is leaving is safely saved, and the state of the world the processor is switching to is correctly restored. The secure world entry to the monitor mode can be achieved by an explicit call via an smc instruction.

\subsection{Address Space Controller}

TrustZone Address Space Controller (TZASC) is an Advanced Microcontroller Bus Architecture (AMBA) compliant SoC peripheral. It allows a TrustZone system to configure security access permissions for each address region. In TrustZone, the access permissions are managed by a group of registers, the access to which must be from the secure world. In addition, TZASC controls data transfer between an ARM processor and Dynamic Memory Controller (DMC). To permit data transfer, it examines whether NS-bit matches the security settings of the memory region. Given a memory region set to secure access only, for example, an attempt to read returns all zeros and that to write has no change to the contents in that region.

\subsection{Memory Management Unit}

An ARM processor also provides MMU to perform the translation of virtual memory addresses to physical addresses. Since TrustZone partitions memory space into secure and normal worlds, a processor with TrustZone enabled provides two separated virtual MMUs which allow each world to map virtual addresses to physical addresses independently.

In the normal world, a process can only access physical memory in the non-secure state. In the secure world, it however can specify how to access physical memory by tuning NS-bit. For example, it could adjust the NS field in the firstlevel page table, and access the memory in either the secure or non-secure state. This flexibility augments a TrustZone system with an ability to efficiently share memory across the worlds.

\section{THREAT MODEL}

TrustShadow shields a trustworthy application from a hostile OS. We consider a completely compromised OS, which means the attacker can execute arbitrary hostile code with system privilege to interfere with the memory and registers of a process. For example, it may read/write any memory in victim process's address space, through either load/store instructions or Direct Memory Access [7, 27], causing memory disclosure and code injection attacks. As another example, OS could modify interrupted process state (e.g., the PC register) during exception handling and resume the execution from an arbitrary instruction to change the program execution's control flow.

In addition, the OS could change victim process's behavior by hijacking system services (e.g., forging system call responses), leading to Iago attacks [11]. Recent study shows that an adversary can infer data by observing a program's page fault patterns [45]. This kind of controlled channel attack is also covered in this work.

Availability is out of scope in this paper. In fact, a compromised OS could simply refuse to boot, or decline the time slices of an HAP to launch Denial-of-Service (DoS) attacks. Side channel attacks such as timing and power analysis are out of scope in this paper. We assume the runtime system running in the TrustZone is trusted. Throughout our design of TrustShadow, we keep its functionality simple and its code 


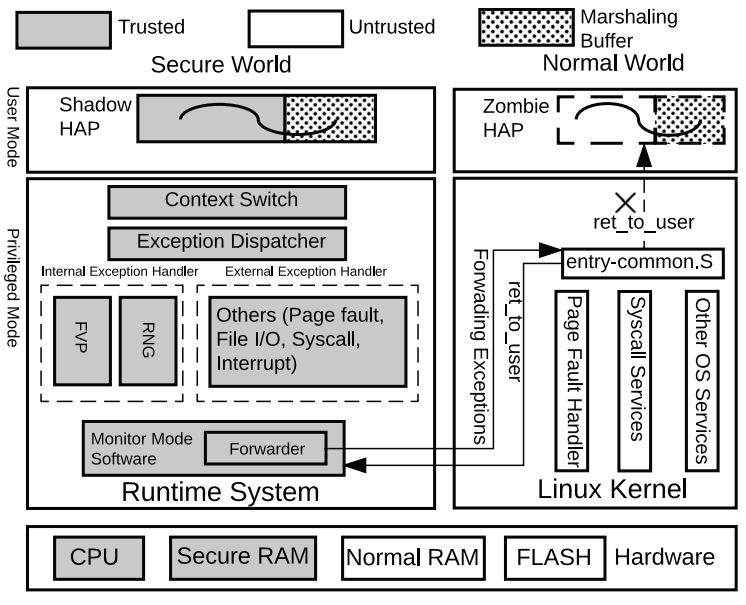

Figure 1: The architecture of TrustShadow.

base minimal. This makes it easier to ensure its correctness through formal verification [41] or manual review.

\section{OVERVIEW}

Figure 1 illustrates the architecture of TrustShadow, where the runtime system and Linux kernel run in the secure and normal world, respectively. Within the secure world, the runtime system shields the execution of a High-Assurance Process (HAP), and all the trusted modules shown in the figure cannot be accessed by the ordinary Linux running in the normal world.

To be resistant to a hostile OS, an application needs to be initialized through a customized system call, which creates a "zombie" HAP and its "shadow" counterpart. In our design, the zombie HAP represents the application running in the normal world. However, it never gets scheduled to run. Rather, TrustShadow runs its "shadow" counterpart residing in the secure world. To support the execution of the shadow HAP, TrustShadow introduces a lightweight runtime system to the secure world.

The runtime system does not provide system services for shadow HAPs. Instead, it intercepts exceptions and forwards them to the Linux OS running in the normal world. In this way, the runtime system can maintain a trusted execution environment for HAPs without introducing a large amount of code to the secure world. To enable crossworld communications, TrustShadow introduces data structure task_shared to share data between the runtime system and the OS. In addition, TrustShadow sets aside data structure task_private to store sensitive metadata for shadow HAPs. In our design, this data structure can only be accessed by the runtime system.

To accommodate the execution of HAPs and coordinate communications across two worlds, the runtime system is designed with various modules (see Figure 1). Serving as the gateway for all exceptions and their returns, the context switch module maintains the CPU hardware context for each HAP, and restores/clears general-purpose registers accordingly. It allows the runtime system to coordinate the execution of an HAP and avoid leaking sensitive data to the OS running in the normal world.

As is shown in Figure 1, the runtime system also im-

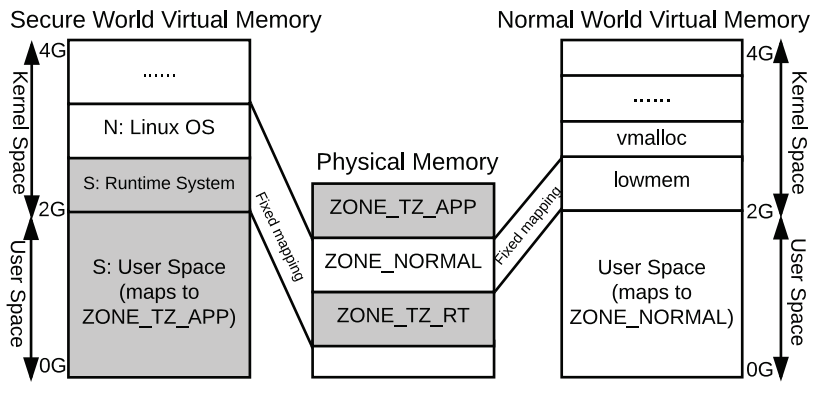

Figure 2: Physical memory partition vs. virtual memory layout.

plements an internal exception handler module - indicated by FVP and RNG. They are designed to handle floating point computation and random number requests locally, for the reasons that cryptographic operation must rely upon trustworthy random number generation, and floating point computation necessarily exposes floating registers if TrustShadow relies upon the Linux OS for handling this exception. In Section 6.5, we describe this internal exception handler in detail.

In our design, the runtime system handles exceptions using three modules, including exception dispatcher, external exception handler, and forwarder. The exception dispatcher is responsible for dispatching exceptions to corresponding handlers. Except for floating point exception and random number requests, this module dispatches all the exceptions to the external exception handler which further redirects the exceptions to the forwarder module. To accommodate exception forwarding in a transparent manner, the forwarder module emulates an exception context for the normal world, pretending that exception is trigger by the zombie HAP. After receiving exceptions, the Linux OS handles them and returns results through task_shared. The external exception handler verifies the return results before reflecting them to the execution environment of the corresponding HAP. In Section 6.2, 6.3 and 6.4, we describe how the three modules coordinate and perform external exception handling.

Since the normal OS does not have the privilege to access a shadow HAP, TrustShadow also introduces a worldshared buffer, indicated as the marshaling buffer in Figure 1. Through this buffer, not only does TrustShadow share the parameters of system calls with the ordinary OS but also retrieves the returns of the system calls. To retrieve the return value of a system call, TrustShadow copies the data in the buffer to the memory region corresponding to the system call, provided that the verifier module marks it valid.

\section{RUNTIME SYSTEM}

In this section, we detail the runtime system illustrated in Figure 1. We begin with memory management for security. Then, we discuss how the aforementioned modules coordinate HAP execution. More specific, we describe how they forward exceptions, handle page faults and intervene system calls. Last, we present internal exception handling.

\subsection{Memory Management}

Here, we describe how we partition physical memory regions, and specify the design of virtual memory system. 
Physical Memory Partition. Using TZASC, TrustShadow creates three distinct physical memory regions. They are non-secure region ZONE_TZ_APP as well as secure regions ZONE_TZ_RT and ZONE_TZ_APP. The non-secure region can be accessed by both the normal and secure worlds, whereas the secure regions have to be accessed through the secure world. In our design, we designate secure region ZONE_TZ_APP for shadow HAPs, ZONE_TZ_RT for the runtime system and nonsecure region ZONE_NORMAL for holding the Linux OS and other ordinary processes. We illustrate these three regions in Figure 2. With the partition above, the runtime, HAPs and Linux OS are all physically isolated, which provides the essential support for safeguarding the HAPs.

Virtual Memory Layout. TrustShadow supports executing legacy Linux code in the secure world. As a result, we design the virtual address of the secure world to follow the same user/kernel memory split as that in the Linux OS. With this design, legacy code can be offloaded to execute in the secure world without any code relocation. In our current design, both Linux OS and the runtime system maintain a $2 \mathrm{G} / 2 \mathrm{G}$ virtual address split, as shown in Figure 2 .

In the kernel space of secure world, in addition to mapping itself to ZONE_TZ_RT, the runtime system maps the physical memory holding the Linux OS (ZONE_NORMAL) in the virtual address space. With this mapping, the runtime system can efficiently locate shared data from the OS (such as task_shared) by adding a corresponding offset.

\subsection{Forwarding Exceptions}

In general, a program is not self-contained. During execution, it might be trapped into the OS (e.g., calling a system service, encountering a page fault or interrupt). In the ARM architecture, system calls are requested by issuing the svc instruction which traps the processor into privileged SVC mode to accomplish the system services. Likewise, other exceptions during execution would trap the processor into the corresponding privileged modes.

As is described in Section 5, except for float point computation and random number generation, the runtime system intercepts exceptions and redirects them to the Linux running in the normal world. Here, we describe how TrustShadow performs exception forwarding.

ARM processors utilize current program status register (cpsr) to hold the current working mode of a processor (e.g., USR or SVC). When an exception is taken, a processor enters the target mode by performing the following operations. First, register pc points to the corresponding offset in the exception vector table. Then, the processor stores the value of previous cpsr to saved program status register (spsr) before setting cpsr to indicate the current working mode (i.e., the target mode). In the ARM architecture, spsr is a banked register and thus each processor mode has its own copy. Based on the value of spsr, an exception handler could get information about the pre-exception processor mode.

Since the monitor mode software can access resources in both worlds, the runtime system can re-produce an exception as follows. Here, we take forwarding a SVC exception as an example. (i) The runtime system sets spsr in monitor mode to represent the target mode (SVC). (ii) It sets the target mode's spsr to represent user mode (USR). (iii) It issues movs instruction to jump to the target exception handler
(0xFFFF0008). Here, movs is an exception return instruction. In addition to jumping to the target address, it copies spsr in the current mode (SVC, which is set in Step i) to cpsr in the target mode. As a result, the OS kernel catches the exception at the correct address (0xFFFF0008) in the right mode (SVC), with spsr indicating that the exception comes from user mode (set in Step ii). We provide a code snippet to demonstrate this implementation in Appendix A. Forwarding other types of exception can be implemented in a similar way.

\subsection{Handling Page Fault}

A page fault is one type of exception resulting from the failure of fetching an instruction or accessing data. In the ARM architecture, a page fault is also called an abort exception, raised by MMU, indicating that the memory accessed does not have a page table entry set properly. After such an exception is taken, an OS invokes its page fault handler which assigns an appropriate physical page and updates the page table entry accordingly. Typically, a page table entry includes the virtual-to-physical address mapping and the access permissions of the virtual memory.

In general, an OS maintains page tables for applications. However, considering that an OS might be hostile and can tamper with the page tables for applications, we isolate these page tables from the OS by placing them in the secure world. The runtime system updates their entries by taking advantage of the page fault handler provided by Linux OS.

To harness the page fault handler, we modify the existing on-demand page fault handing mechanism in Linux. In particular, we hook the page fault handler so that it can store the context of page fault handling in the aforementioned shared memory, task_shared ${ }^{2}$. After retrieving the updating information and before installing a page table entry, the runtime system validated the returned information. In the following, we provide more details on this procedure.

\subsubsection{Basic Page Table Update}

A page fault occurs in various situations, and TrustShadow handles page faults differently. The most simple situation is accessing anonymous memory, in which case the page table entries in the Linux OS and the runtime system point to the same secure page $\mathbb{S}$. Besides duplicating the page table entry retrieved from task_shared, the run time system must first make sure that the mapped page is within the memory region defined in ZONE_TZ_APP. We illustrate this basic page fault handling in Figure 3a.

\subsubsection{Page Table Update with Integrity Check}

Different from anonymous memory, accessing memory backed by a file needs assistance from the OS to load the file contents to memory. As the OS may be hostile, besides ensuring that the translated pages are within the range of ZONE_TZ_APP, the runtime system also verifies the integrity of loaded contents. This section describes page fault handling on the memory regions backed by executable images.

We take loading a code page as an example in Figure $3 \mathrm{~b}$. When a prefetch abort happens, the Linux page fault handler will eventually call do_read_fault, which lo-

\footnotetext{
${ }^{2}$ In our design, task_shared carries the updated page table entry value (which contains the address of the translated physical memory page), the influenced virtual address, and additional contextual information.
} 


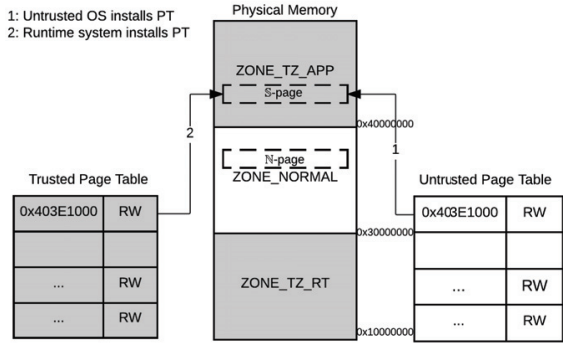

(a) Basic Page Table Update.

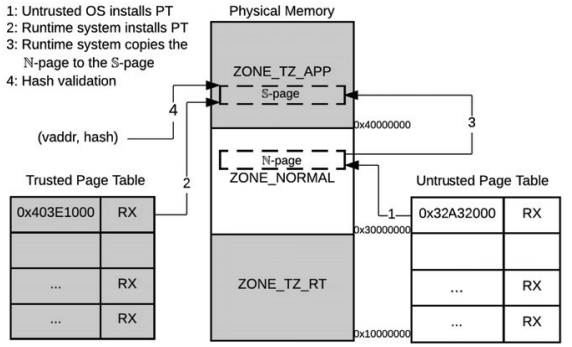

(b) Page Table Update with Integrity Checking.

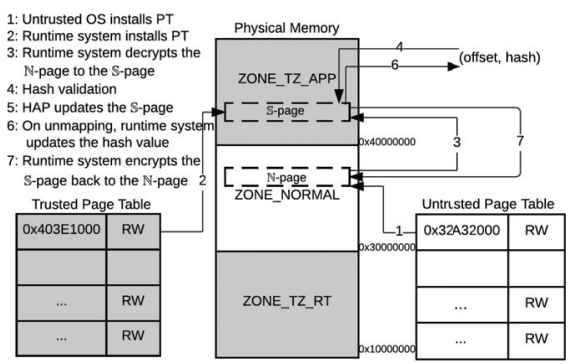

(c) Page Table Update for Protected Files.

Figure 3: Page Table Update.

cates the physical page $\mathbb{N}$ caching the corresponding code page (Step 1). In this context, a new secure world page $\mathbb{S}$ from ZONE_TZ_APP is allocated, and the physical addresses of both $\mathbb{N}$ and $\mathbb{S}$ pages are saved in task_shared. With this shared information, the runtime system first ensures that $\mathbb{S}$ page is actually a fresh page from ZONE_TZ_APP. Then, the runtime system installs a new page table entry in the trusted page table (Step 2), copies the $\mathbb{N}$ page to the $\mathbb{S}$ page (Step 3) and verifies the integrity of the copied page (Step 4). Note that verification is performed on $\mathbb{S}$ pages, therefore, TrustShadow is resilient to TOCTTOU (Time Of Check To Time Of Use) attacks.

The described page table update with integrity check is the low level primitive for ensuring load time program integrity. TrustShadow enforces such checking on all the memory segments of type PT_LOAD in the ELF program images, including executable and dynamic libraries. In the following, we provide details on verifying the integrity of program images.

Verifying Executable Integrity. The Runtime system maintains a list of hash values in the format of (vaddr, hash), which is initialized according to the bundled manifest (see Section 6.6). Once a page fault occurs in the covered range, the runtime system installs a secure page table entry as mentioned above. If validation is failed, the runtime system immediately terminates the process by sending an _exit system call to the OS. We note that such validation is a one-time effort, so it does not influence execution performance at run time when the program is warmed up.

Verifying Shared Library Integrity. Different from executables, shared libraries are position independent. To verify pages loaded for shared libraries, the runtime system maintains a system wide (offset, hash) list for all shared libraries frequently used. When a shared library image is mapped in the address space, the runtime system obtains the loaded base address baseAddr by monitoring the return value of the mmap system call. Then, the integrity of the loaded page is verified at address (baseAddr + offset).

\subsubsection{Page Table Update for Protected Files}

This section describes page fault handling of data files. Different from executable image, the purpose of protecting data file is to prevent OS from accessing clear-text contents. Therefore, the runtime system further employs encryption technique in handling this type of page fault. TrustShadow allows developers to differentiate data files based on their the sensitivity levels. Only sensitive files that are specified in a manifest bundled with the application (see Section 6.6 for details) are protected.

Before elaborating page fault handling when accessing protected files, we first describe how TrustShadow manages them at high level. All the operations accessing these files are transparently transformed into memory mapped I/O. To correctly map file descriptor offsets to virtual addresses, preceding pages of a file are reserved for meta-data. This includes the real file length, time stamp of the last access, along with hash values of the real data pages. These preceding meta pages are protected by a per-application AES key that is provided by the manifest.

As shown in Figure 3c, when accessing a non-present page of a protected file, the runtime system decrypts the ciphertext $\mathbb{N}$ page loaded by the OS, and writes it into a secure $\mathbb{S}$ page (Step 3), which is also allocated by the OS and verified by the runtime system. After that, the hash value of the page is calculated and validated (Step 4). When unmapping this page, the runtime system recalculates and stores the hash value of the updated $\mathbb{S}$ page (Step 6), and then encrypts it into the original $\mathbb{N}$ page (Step 7), which is finally written to the permanent storage by the OS. The encryption key, again, is the per-application AES key that comes with the manifest.

\subsection{Intervening System Calls}

Two problems are raised when a system call is forwarded to the OS. First, due to isolation, the OS kernel cannot access the address space of a shadow HAP, while some system call services rely on input data from user space. Second, the results returned by the OS are not trusted, which may lead to potential attacks. The runtime system coordinates the intervention between an HAP and the OS, provides the OS with essential service request data, and verifies the responses from an untrusted OS. For critical system services that cannot be served by the OS (e.g., random number generator), the runtime system implements them inside the secure world, which is discussed in Section 6.5.

\subsubsection{Adapting System Calls}

Memory isolation changes the way that the OS manages and accesses the memory of an HAP. Without the runtime system acting as an intermediator, it is impossible for the OS to access application data containing system call requests. We follow existing marshaling techniques available on x86 platform, in which system call parameters are adapted in a world-shared buffer. This allows the OS to have temporary 
access to system call parameters. Besides this, there still remains challenges that are specific to our design. This section briefly reviews existing marshaling technique, and then describes our specifics.

Parameter Marshaling. Most system call parameters are scalar in that they contain values instead of pointers to memory, for instance, close, getpid, and _exit. The runtime system forwards them directly without any modification.

However, complex system calls allow a process to pass in a pointer so the kernel can read data from or write result to a user space buffer. For example, the open system call passes in a buffer containing the file path as a pointer. As the OS cannot access user space data, the runtime system marshals them into a world-shared buffer, and adjusts the parameters accordingly. The system call service works on the marshaling buffer. After it completes, the runtime system copies back the results into the original user buffer if necessary. More complex system calls, such as ioctl and fcntl, have different behaviors according to subcommands. A marshaling code for each request/cmd must be prepared separately according to the specifications of the subcommands.

Signal. In signal handling, a signal delivery allows an untrusted OS to resume user space code at arbitrary location, thus compromising control flow integrity of a shadow HAP. In addition, setup_frame needs to manipulate the process's stack to craft signal information and return code, while the OS has no privilege to do so.

TrustShadow addresses these problems by both hacking the OS code and supporting in the runtime system. Specifically, when a signal is registered, the runtime system inserts the handler address into the task_private structure of the shadow HAP. When a signal is caught by the OS, a reserved page in the marshaling buffer is used by setup_frame to set up a separate user mode stack specifically for signal handling. At the same time, the intended return address for signal handler is placed in task_shared. When the runtime system resumes, it first verifies that the address has been registered and that the pretcode on the signal stack is correct $^{3}$. If so, the signal stack is copied to an unused virtual address backed by a secure page ${ }^{4}$. Then the hardware context of the normal control flow is saved in a temporary structure in task_private, and is replaced with the signal's hardware context. When the signal handler returns by issuing the rt_sigreturn system call, the hardware context of the normal control flow is restored.

Futex. Fast userspace mutex (futex) is another interesting kernel service that conflicts with process isolation. In Linux, a futex is identified by a four-bytes memory shared among processes or threads. It acts as a building block for many higher-level locking abstractions such as semaphores, POSIX mutexes, and barriers. If a thread fails to acquire a lock, it passes the lock's address along with its current value to a futex wait operation. This futex operation will block the thread if and only if the value in lock's address still matches the value it received. The blocked thread resumes

\footnotetext{
${ }^{3}$ pretcode points to a piece of code calling the rt_sigreturn system call on sigpage. This piece of code is common to all the processes.

${ }^{4}$ TrustShadow reserves configurable number of secure pages specifically for this purpose.
}

when another thread releases the lock by issuing a futex wake operation, which unblocks all the threads waiting on a specific lock. Obviously, the futex system call needs to read the value of the lock which is in the user space of an HAP.

We observe that a thread never waits for more than one futex at a time ${ }^{5}$. Therefore, we hack the futex system call to always read from a fixed memory location in the marshaling buffer. Each time a futex wait operation is issued, the runtime system synchronizes the current futex value to that fixed address. In TrustShadow, we further handle a futex shared across processes by maintaining a system wide map that keeps physical addresses of involved memories. The runtime system queries this map to synchronize futex updates to different processes.

\subsubsection{Defeating Iago Attack}

As disclosed in [11], a compromised OS could subvert an HAP by manipulating the return values of system calls, thus leading to Iago attacks. For example, when an HAP requests a new memory region through the mmap system call, it expects that the returned region is disjoint with any existing mapping in the process's address space. However, a compromised OS could return an address that overlaps with the process's stack. Without proper checking on the return values, the following write on the new region would smash the stack and the process can be coerced into executing a return-oriented program [10].

With the runtime system sitting in-between the shadow HAP and the untrusted OS, it is straightforward to address known Iago attacks by interposing the system call interface and checking their results. For a known Iago attack, we need a specification for that particular system service. Here, we take the mmap system call as an example. Every return address of the mmap or brk system system call is compared with the current memory mapping. If an overlap is found, the HAP is immediately killed. The runtime system collects current memory mapping in three ways. First, the range of stack is obtained from current sp, because the stack spans from sp to the top of user space virtual memory. Second, heap limit can be monitored by examining return values of the brk system calls. Finally, the return value of each successful mmap/munmap system call is recorded.

\subsection{Internal Exception Handling}

In this section, we list security-critical exceptions that are handled directly inside the runtime system. Forwarding them to the OS would leak user data or lead to security breach.

Floating Point Computation. ARM architecture supports hardware floating point calculation by Vector Floating-Point (VFP) architecture extension. VFP introduces a set of registers and instructions specific for floating point calculations. The access to them is controlled by a privileged register FPEXC. In Linux, when a program accesses VFP for the first time, an UNDEFINED exception is raised and the kernel is responsible for enabling VFP support for this program. To support multiple processes accessing VFP concurrently, the kernel maintains a VFP context for each process in its kernel stack. This design obviously leaks user data contained in VFP registers to kernel. In TrustShadow, the

\footnotetext{
${ }^{5} \mathrm{~A}$ blocked thread can never issue another futex wait operation.
} 


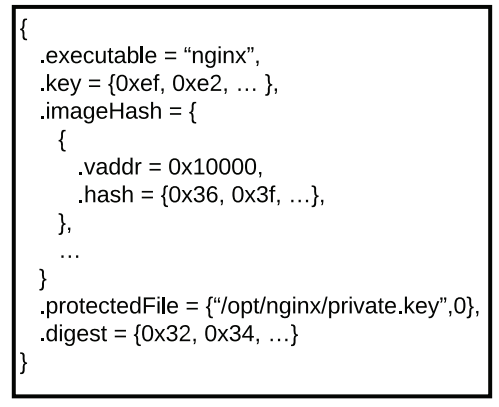

Figure 4: A sample manifest. Note that protectedFile specifies the file names that TrustShadow needs to protect; digest indicates the signature of this manifest.

runtime system duplicates the code handling VFP from the Linux OS. More specifically, the runtime system maintains a VFP context in the secure memory for each HAP that requires VFP calculation, and clears VFP registers whenever switching to the ordinary OS.

Random Number Generator. The Linux pseudoRandom Number Generator (LRNG) is the main source of randomness for many cryptographic applications, such as OpenSSL. Linux provides LRNG service by exposing /dev/(u)random devices to applications. Since using weak random values is a catastrophe for cryptographic systems, and an untrusted OS should not know the key materials used in the application, TrustShadow provides a trusted RNG service in the secure world. Specifically, the runtime system maintains a list of file descriptors that correspond to opened /dev/(u)random devices. Read operations on these descriptors are intercepted such that trusted random values are directly provided. The runtime system readily utilizes the on-board hardware random number generator RNG4 to generate strong random numbers.

\subsection{Manifest Design}

As mentioned in prior sections, each HAP is bundled with a manifest that provides metadata for the security features. We design a manifest to contain the following - a perapplication secret key, the integrity metadata of the application (i.e., the (vaddr, hash) list), and a list of file names that should be cryptographically protected.

Since the manifest is stored on a local persistent storage which can be accessed by the OS, we design two mechanisms to ensure its security. First, we encrypt the per-application secret key using a per-device public key. Therefore, only the runtime system which has access to the per-device private key is able to decrypt it. Second, to ensure the integrity of the manifest, we append a digital signature calculated on the content of the manifest using a per-device private key. In a real deployment, we note that per-device public/private key pairs used for encryption and signature should be separated. In the presentation of this paper, we refer to them as a single key pair for simplicity. Figure 4 shows a C-data-structureequivalent of a sample manifest we used to safeguard the Nginx web server.

\section{IMPLEMENTATION}

We have implemented TrustShadow on a Freescale

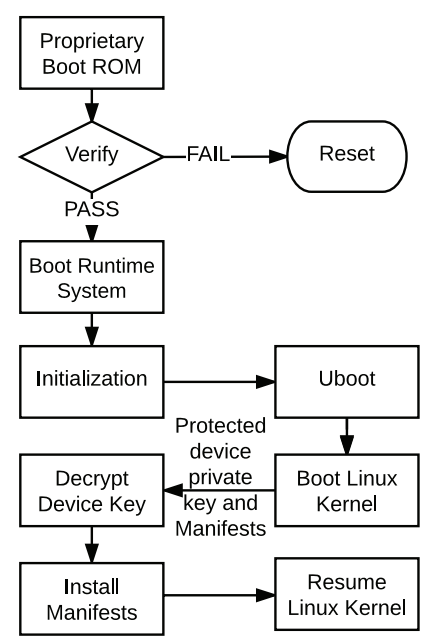

Figure 5: The boot sequence of TrustShadow. The components on the left and right indicate the operations in the secure and normal world, respectively.

i.MX6q ARM development board that integrates an ARM Cortex-A9 MPCore processor, 1GB DDR3 DRAM and $256 \mathrm{~KB}$ iRAM. As is discussed in the section above, TrustShadow involves operations on both the normal and secure worlds. In this section, we therefore describe our implementation details in turn.

\subsection{Normal World}

In the normal world, we made the following changes to a Linux OS distribution with kernel version 3.18.24. (i) We added kernel parameter tz_mem=size@start which indicates the memory region used for HAPs, i.e., ZONE_TZ_APP. (ii) We changed zone-based allocator to ensure that the pages designated to shadow HAPs must be from the memory region indicated by $t z \_m e m=s i z e @ s t a r t$. (iii) We added a tz flag to task_struct in order to make the OS capable of distinguishing HAPs. (iv) We implemented a new system call tz_execve in order to start an HAP in Linux. (v) We changed the control flow of ret_to_user and ret_fast_syscall, so that the Linux OS can pass the execution back to a corresponding shadow HAP instead of a zombie HAP. (vi) We hooked the page fault handler so that it can prepare page table update information for the runtime system. (vii) We modified the code handling signals in order to set up a signal stack in the marshaling buffer and make it ready for an HAP. In total, these changes introduce about 300 LOC to the Linux kernel.

\subsection{Secure World}

In the secure world, we implemented the aforementioned runtime system with about $4.5 \mathrm{~K}$ LOC of ANSI C and $0.8 \mathrm{~K}$ LOC of assembly. In addition, we implemented a secure boot mechanism to guarantee the integrity of TrustShadow. Figure 5 describes how we implemented a secure boot for TrustShadow.

Using High Assurance Boot (HAB), a proprietary boot ROM first loads the image of the runtime system. Then, it performs a verification and examines the integrity of the image. After passing the integrity check, the runtime system starts, using TZASC to configure the access policy of mem- 


\begin{tabular}{|l|c|c|c|c|c|}
\hline & \multicolumn{2}{|c|}{ Latency $(\mu \mathrm{s})$} & \multicolumn{3}{c|}{ Overhead } \\
\hline Test case & Linux & $\begin{array}{c}\text { Trust } \\
\text { Shadow }\end{array}$ & $\begin{array}{c}\text { Trust } \\
\text { Shadow }\end{array}$ & InkTag & $\begin{array}{c}\text { Virtual } \\
\text { Ghost }\end{array}$ \\
\hline null syscall & 0.7989 & 1.6048 & $2.01 \mathrm{x}$ & $55.80 \mathrm{x}$ & $3.90 \mathrm{x}$ \\
\hline open/close & 29.2168 & 40.7886 & $1.40 \mathrm{x}$ & $4.83 \mathrm{x}$ & $7.95 \mathrm{x}$ \\
\hline mmap (64m) & 559.0000 & 784.0000 & $1.40 \mathrm{x}$ & $4.70 \mathrm{x}$ & $9.94 \mathrm{x}$ \\
\hline pagefault & 4.7989 & 7.9764 & $1.66 \mathrm{x}$ & $1.15 \mathrm{x}$ & $7.50 \mathrm{x}$ \\
\hline $\begin{array}{l}\text { signal handler } \\
\text { install }\end{array}$ & 1.6257 & 3.8294 & $2.36 \mathrm{x}$ & $3.24 \mathrm{x}$ & - \\
\hline $\begin{array}{l}\text { signal handler } \\
\text { delivery }\end{array}$ & 51.6111 & 57.0349 & $1.11 \mathrm{x}$ & $1.61 \mathrm{x}$ & - \\
\hline fork+exit & 987.0000 & 2328.6000 & $2.36 \mathrm{x}$ & $4.40 \mathrm{x}$ & $5.74 \mathrm{x}$ \\
\hline fork+exec & 1060.3333 & 2509.0000 & $2.37 \mathrm{x}$ & $4.20 \mathrm{x}$ & $3.04 \mathrm{x}$ \\
\hline select (200fd) & 15.0707 & 18.8649 & $1.25 \mathrm{x}$ & $3.40 \mathrm{x}$ & - \\
\hline ctxsw 2p/0k & 30.3700 & 32.7100 & $1.08 \mathrm{x}$ & - & $1.41 \mathrm{x}$ \\
\hline
\end{tabular}

Table 1: LMbench micro-benchmark results.

ory regions ZONE_TZ_RT, ZONE_NORMAL, and ZONE_TZ_APP. To guarantee the policy cannot be maliciously altered, the runtime locks the configuration. As a result, further modifications to the policy requires system reboot.

After the success of initialization, the runtime system loads uboot binary [44] which further boots the Linux system implemented above. The Linux system runs in the normal world where it retrieves the manifest as well as the public/private key pair stored on the persistent storage. Note that, our implementation encrypts the public/private key pair in advance using the 256-bit Zeroizable Master Key (ZMK) stored on Freescale i.MX6q board. This ensures the key pair is not disclosed to the Linux in plaintext. We believe this implementation is a common practice for many device manufacturers [38].

To facilitate the secure boot, the Linux system passes the manifest and public/private key pair to the runtime system which further decrypts the key pair and installs the manifest. With this process completion, the runtime passes the execution back to the Linux system.

\section{EVALUATION}

In this section, we evaluate TrustShadow by conducting extensive experiments. Using microbenchmarks, we first explore the impact of TrustShadow upon primitive OS operations. Second, we quantify the overhead of I/O operations imposed by TrustShadow. Last, we run real world applications and study the overall performance overhead introduced by our system. We conducted the aforementioned experiments on a Freescale i.MX6q board running both native Linux and our TrustShadow. We treated the performance observed from native Linux as our baseline and compared it with that observed from TrustShadow.

\subsection{Microbenchmarks}

Using LMBench [36], we study the overhead imposed to basic OS operations. More specifically, we ran various system services present in Table 1 against both native Linux and TrustShadow. To minimize the noise involved during our experiment, we ran each benchmark with 1,000 iterations and took the average as our measures. We also compared the overhead imposed by TrustShadow with that introduced by InkTag [26] and VirtualGhost [16]. This is because both share the same goal with our system although they are designed specifically for x86 architecture and not applicable to IoT devices typically embedded with ARM processors.

Table 1 shows the results indicating the overhead that

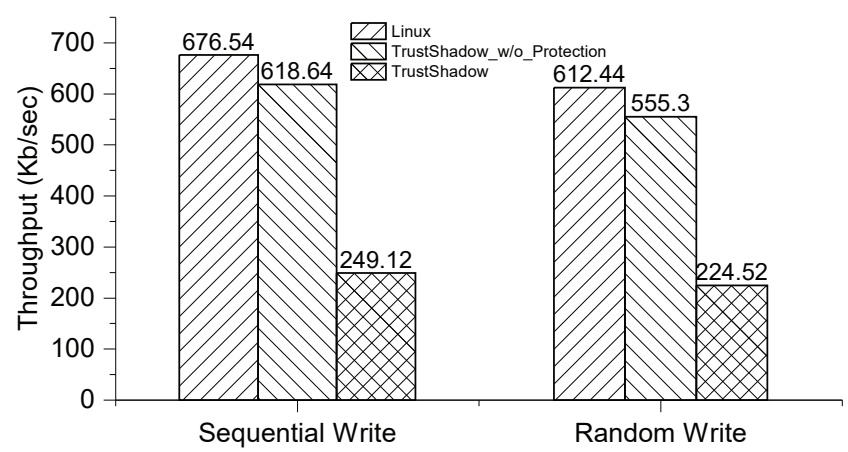

Figure 6: File I/O performance as measured by sequential and random write.

TrustShadow imposes to various system services. In addition, it presents the overhead introduced by InkTag and VirtualGhost. Note that we did not run experiments on these two systems. Rather, we obtained their overhead measures from the articles previously published in $[26,16]$.

First, we observe that TrustShadow introduces considerable overheads to individual operations. Most notably are fork+exit, fork+exec and signal handler install, all of which increase overhead by about $2.36 \mathrm{x}$. The high overhead introduced by the first two services is mainly due to the fact that TrustShadow optimizes the OS to populate all the marshaling buffer in one go when creating a new thread. And, the high overhead imposed by signal handler install results from copying a signal stack from the page in the normal world to one in the secure world.

Second, across most test cases shown in Table 1, we observe that the overhead imposed by TrustShadow are relatively lower than that introduced by InkTag and VirtualGhost. The reason is, InkTag and VirtualGhost require additional CPU cycles to communicate with Virtual Machine (VM) or execute the code instrumented to kernel, whereas TrustShadow does not rely on VM nor instrument large amount of code to kernel. Compared with InkTag, we also observe that TrustShadow imposes high overhead to system service pagefault. This is because TrustShadow needs to perform additional page copy or zeroization, which is not required for InkTag.

While the overhead shown in the table appears large, it should be noted that, this does not imply that TrustShadow jeopardizes the performance of applications under protection. In fact, applications are significantly less sensitive to system services. As we will show later in the section, TrustShadow imposes only negligible performance overhead to application execution.

\subsection{File Operations}

To quantify the overhead imposed to I/O throughput, we conducted an experiment by using Sysbench [29] in different modes. As is discussed earlier, Trust Shadow allows developers to designate whether or not to protect a particular file. Thus, we did this experiment with and without file protection enabled.

We prepared 128 files, each of which has $8 \mathrm{Mb}$, and tested both sequential write and random write. To minimize cache effects and best reflect the actual I/O performance, we con- 


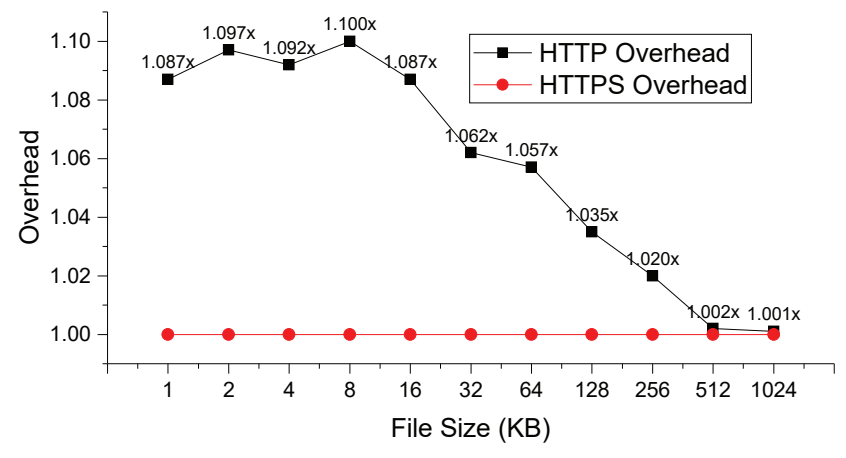

Figure 7: Throughput overhead imposed by TrustShadow across HTML responses in different sizes.

ducted experiments with write-through mode, and forced a call to fsync() after each write operation.

Figure 6 shows the results. Because caching is disabled, sequential write did not exhibit significant advantage over random write. In both cases, the slowdown introduced by TrustShadow without file protection is about $1.09 \mathrm{x}$, while the slowdown by TrustShadow with file protection is about 2.71x. This is due to the fact that TrustShadow with file protection enabled involves heavy encryption and hashing computations when it synchronizes pages to persistent storage. Note that the difference between TrustShadow with and without file protection solely results from cryptographic operations. As a result, employing a more efficient cryptographic engine is a straightforward way of improving file I/O performance.

\subsection{Embedded Web Server}

To study the impact of TrustShadow upon real-world applications, we mimic an embedded web server running on an IoT device. More specifically, we ran the Nginx web server with version 1.9.15 on our testbed against both native Linux and TrustShadow. We configured Nginx to respond with HTML file in different sizes through both HTTP and HTTPs. To quantify the server throughput, we utilized the Apache benchmark [1] on another machine to connect to the server. We configured the benchmark program to create 10 connections simultaneously sending 10,000 HTTP requests. This experiment setting allows us to overwhelm the server and thus compare the throughput variation from the viewpoint of an end user.

Figure 7 shows the throughput overhead of Nginx. Regarding to the requests through HTTP, we observe that, TrustShadow downgrades the server throughput by about $6 \% \sim 10 \%$ when a client requests a file in a relatively small size. However, this throughput downgrade is alleviated when the requested file increases. As is shown in Figure 7 , the server throughput drops only by $2 \%$ when a client requests a file with a size more than $256 \mathrm{~KB}$. Regarding latency, we measured the $95 \%$ percentile in each experiment, and found almost no latency overhead. The raw HTTP performance measurements can be found in Appendix B. In addition, we discover TrustShadow introduces nearly no overhead regardless of whether the size of the file requested varies when HTTP traffic is carried through TLS. The reason behind these observations is that the overhead imposed by TrustShadow is overwhelmed by intensive I/O and computationally intensive cryptographic operations, respectively.

\subsection{Data Analytics}

We also evaluate the performance overhead when performing data analysis using machine learning techniques. This kind of applications is popular for many edge computing platforms. To evaluate the impact of TrustShadow upon such applications, we chose two data analytic applications to emulate face recognition and image classification. To be more specific, we ran an DLIB image classification program [28] powered by deep neural networks against 60,000 images from the MNIST database [30], as well as a face recognition program against 10 images with human faces from the DLIB toolkits.

In the first experiment, we observed that TrustShadow classifies 60,000 images in 998 seconds, which is only 4 seconds higher than performing the same classification on native Linux. In the second experiment, we observed that TrustShadow recognizes faces in 163.271 seconds, whereas native Linux finishes the same task in 162.591 seconds. The observations from both experiments again indicate TrustShadow introduces only negligible overhead to IoT devices.

\section{DISCUSSION AND FUTURE WORK}

In this section, we first summarize how TrustShadow defeats OS level attacks to an HAP. Then, we analyze the security of TrustShadow, quantify its TCB, and discuss the remaining attack surface. Finally, we discuss future work.

HAP Security. TrustShadow protects an application from three aspects. (1) With a mechanism to verify the integrity of a program image, an attacker cannot manipulate application code/data at load time. (2) With the isolation of resources and introspection mechanism, an attacker cannot interfere with HAP execution at run time. The only user memory that the OS can access is the marshaling buffer. (3) With a cryptographic mechanism encrypting files and signing meta data, attackers can no longer read a file under protection or make any modification to it.

Runtime System Security. The protection above is established on the basis of the correctness and robustness of the runtime system. Our design guarantees the security of the runtime system from three aspects. First, our design ensures the integrity of the runtime system at load time because the hash of the verification public key is burned in the chip's fuses and HAB uses this key to verify the signature of the runtime system image before loading it. Second, our design reduces the attack surface of the runtime system because the runtime system itself is loaded into a secure physical region which the ordinary OS cannot manipulate. Third, our design raises the bar for exploiting the runtime system. This is due to three reasons. (1) An application must undergo critical security reviews before being authorized to run as an HAP (i.e., providing it with a manifest with manufacture signature). (2) Even if an HAP has vulnerabilities that may be exploited to execute arbitrary code, it only runs with user privilege. (3) The interface exposed by the runtime system is narrow, because it simply forwards most exceptions to the OS. Also, the small code base of runtime system makes it possible for formal verification. 
TCB Size. To demonstrate the security of TrustShadow quantitatively, we identify the TCB of our system, and compare its size with $\mathrm{x} 86$ alternatives.

Ultimately, all the code in the user's TCB must be trusted. Therefore, like all the other works in this line, user application is included in the TCB. The size of user application is highly dependent on its functionality and complexity. We rely on code review to achieve trust for this part of TCB.

The runtime system maintains the execution environment for an HAP, and thus must be included in the TCB. As mentioned earlier, our runtime system has only about $5.3 \mathrm{~K}$ LOC, which we believe is small enough for manual review or formal verification. In comparison, previous x 86 works have their own privileged code that must be trusted. Hypervisorbased solutions $[13,12,46,26]$ include the whole hypervisor in its TCB, bloating their TCBs by several hundreds of thousands of lines of code. Although thinner hypervisors exist [33], we are not aware of any similar system built on top of them. Haven [6] includes LibOS, a large subset of Windows in its TCB, resulting a TCB of millions LOC. VirtualGhost [16] includes about 5.3K LOC for their run-time system and LLVM passes. This is the only solution that has comparable TCB with TrustShadow.

Remaining Attack Surface. To minimizes TCB, the runtime system does not implement system services itself, but relies on the OS. With full control of process scheduling, the OS can easily launch DoS attacks to an HAP. Similarly, to start an HAP, the OS may choose to invoke the normal execve system call instead of tz_execve. However, the process is executed in the normal world, so it cannot access cryptographically protected files.

Another concern is the manipulation of manifest files. If a manifest file can be forged, integrity checking of the corresponding executable image is bypassed. As a result, arbitrary code can be loaded in the secure world. We address this problem by signing the manifests using a per-device private key. When a vulnerable program is updated, the corresponding manifest should be updated as well. A roll-back attack happens when an attacker executes the vulnerable version of the program with an older manifest. To prevent this from happening, one of our future work is to add a version number field in the manifest, and periodically communicate a list showing the updated version numbers of trusted programs between the runtime system and a remote server.

Last but not least, side channel attacks have been developed to extract information across processes in an OS, or even virtual machines [47, 49, 24, 31]. For example, in [24], the authors introduced a cache storage channel that exploits the inconsistence of cache and physical memory data, and infers victim's behaviors in TrustZone by checking if the constructed inconsistence has been destroyed. TrustShadow's current design may be subject to this line of side channel attacks. However, we can adopt existing techniques to mitigate such attacks. For example, contemporary cryptographic libraries such as OpenSSL have already been designed to resist some side channel attacks [40].

Future Work. Many IoT devices, such as cyber-physical systems, are sometimes deployed in an unmonitored environment. As a result, secret data stored in the DRAM chip is subject to inexpensive physical attacks, such as cold-boot attack [25, 37], bus monitoring [23], and DMA attacks [7, $27]$. In $[14,48]$, the authors proposed to process confiden- tial data within SoC components such as cache and iRAM, because it is considered much harder to compromise a SoC component. In TrustShadow, we can simply configure the region ZONE_TZ_APP to be within the range of iRAM to make HAPs immune to physical attacks.

We have tested this idea on our experiment board, which integrates a $256 \mathrm{~KB}$ iRAM. We successfully ran a small program that generates a 2048-bit RSA key-pair with OpenSSL. As the capacity of iRAM is usually limited, this enhanced security feature could only be activated for low footprint programs. One of our future work is to "extend" iRAM by introducing another level of virtual memory - utilizing DRAM as a backup storage for encrypted iRAM pages.

Although hypervisor-based solutions are not applicable to shield trusted applications in ARM platform as discussed in Section 2, the idea of cloaking memory with a privileged layer has long been studied. With a hypervisor, trusted applications can run in the normal world, which could greatly reduce the risk in the presence of a vulnerable runtime system. While TrustZone is not designed to provide features such as shadow page table that a standard hypervisor could provide, some existing work, such as TZ-RKP [5] and Sprobes [22], has the potential to emulate these features in the secure world. Integrating the technique of across worlds hypervision with existing shielding mechanisms remains another topic for our future work.

\section{CONCLUSION}

In this paper, we have presented TrustShadow that utilizes a carefully designed runtime system to shield applications running on multi-programming IoT devices. With TrustShadow, security-critical applications on these devices can be comprehensively protected even in the face of total OS compromise. Unlike techniques previously proposed, the design of TrustShadow does not require modification to applications. As a result, security can be guaranteed without the requirement of re-engineering applications. Since TrustShadow imposes only negligible - and occasionally moderate - overhead to IoT devices, the protection of an application can be achieved in a lightweight manner. With an increasing number of IoT devices developed, we expect the design of TrustShadow could inspire more research in the area of IoT computing.

\section{ACKNOWLEDGMENTS}

We would like to thank the anonymous reviewers for their insightful feedback and our shepherd, Ardalan Amiri Sani, for his valuable comments on revision of this paper. This work was supported by U.S. Army Research Office award W911NF-13-1-0421 (MURI), NSF under Grant No. CNS1422594, CNS-1505664, CNS-1634441, CNS-1422355, CNS1408880, SBE-1422215, and the Penn State Institute for CyberScience (ICS) Seed Funding Initiative grant. Any opinions, findings, and conclusions or recommendations expressed in this material are those of the authors and do not necessarily reflect the views of the National Science Foundation and U.S. Army Research Office.

\section{REFERENCES}

[1] Apache Software Foundation. Apache HTTP server benchmarking tool. http://httpd.apache.org/docs/2.4/programs/ab.html. 
[2] ARM Architecture Group. Virtualization Extensions Architecture Specification, 2010. https://www.arm.com/products/processors/ technologies/virtualization-extensions.php.

[3] ARM Architecture Group. ARMv8-M Architecture Simplifies Security for Smart Embedded Devices, 2015. https://www.arm.com/about/newsroom/armv8m-architecture-simplifies-security-for-smartembedded-devices.php.

[4] ARM Ltd. Security technology building a secure system using trustzone technology (white paper). 2009.

[5] A. M. Azab, P. Ning, J. Shah, Q. Chen, R. Bhutkar, G. Ganesh, J. Ma, and W. Shen. Hypervision across worlds: Real-time kernel protection from the arm trustzone secure world. In $A C M C C S^{\prime} 14$, 2014, pages 90-102. ACM, 2014.

[6] A. Baumann, M. Peinado, and G. Hunt. Shielding applications from an untrusted cloud with haven. In OSDI'14, 2014, pages 267-283, Broomfield, CO, Oct. 2014. USENIX Association.

[7] M. Becher, M. Dornseif, and C. Klein. Firewire: All your memory are belong to us. In 6th Annual CanSecWest Conference, 2005.

[8] F. Bonomi, R. Milito, P. Natarajan, and J. Zhu. Fog computing: A platform for internet of things and analytics. In Big Data and Internet of Things: A Roadmap for Smart Environments, pages 169-186. Springer, 2014.

[9] F. Bonomi, R. Milito, J. Zhu, and S. Addepalli. Fog computing and its role in the internet of things. In Proceedings of the First Edition of the MCC Workshop on Mobile Cloud Computing, MCC '12, pages 13-16, New York, NY, USA, 2012. ACM.

[10] S. Checkoway, L. Davi, A. Dmitrienko, A.-R. Sadeghi, H. Shacham, and M. Winandy. Return-oriented programming without returns. In $A C M C C S^{\prime} 10,2010$, pages 559-572. ACM, 2010.

[11] S. Checkoway and H. Shacham. Iago attacks: Why the system call api is a bad untrusted rpc interface. In ASPLOS'13, 2013, pages 253-264, New York, NY, USA, 2013. ACM.

[12] H. Chen, F. Zhang, C. Chen, Z. Yang, R. Chen, B. Zang, P.-c. Yew, and W. Mao. Tamper-resistant execution in an untrusted operating system using a virtual machine monitor. Technical Report FDUPPITR-2007-0801, Parallel Processing Institute, Fudan University, August 2007.

[13] X. Chen, T. Garfinkel, E. C. Lewis, P. Subrahmanyam, C. A. Waldspurger, D. Boneh, J. Dwoskin, and D. R. Ports. Overshadow: A virtualization-based approach to retrofitting protection in commodity operating systems. In $A S P L O S^{\prime} 08$, 2008, pages 2-13, New York, NY, USA, 2008. ACM.

[14] P. Colp, J. Zhang, J. Gleeson, S. Suneja, E. de Lara, H. Raj, S. Saroiu, and A. Wolman. Protecting data on smartphones and tablets from memory attacks. In ASPLOS'15, 2015, pages 177-189, New York, NY, USA, 2015. ACM.

[15] N. Constant, D. Borthakur, M. Abtahi, H. Dubey, and K. Mankodiya. Fog-assisted wiot: A smart fog gateway for end-to-end analytics in wearable internet of things. CoRR, abs/1701.08680, 2017.

[16] J. Criswell, N. Dautenhahn, and V. Adve. Virtual ghost: Protecting applications from hostile operating systems. In $A S P L O S^{\prime} 14$, 2014, pages 81-96, New York, NY, USA, 2014. ACM.

[17] CVEdetails.com. Vmware: Vulnerability statistics. http: //www.cvedetails.com/vendor/252/Vmware.html.

[18] CVEdetails.com. Xen: Vulnerability statistics. http://www.cvedetails.com/vendor/6276/XEN.html.

[19] Eric Brown. First linux-based 3d printers hit the market. http://hackerboards.com/first-linux-based3d-printers-hit-the-market/.

[20] Felix Baum. Why you don't necessarily need a hypervisor, 2014. http://embedded-computing.com/guest-blogs/whyyou-dont-necessarily-need-a-hypervisor/.

[21] T. Garfinkel, B. Pfaff, J. Chow, M. Rosenblum, and D. Boneh. Terra: A virtual machine-based platform for trusted computing. In SOSP'03, 2003, pages 193-206, New York, NY, USA, 2003. ACM.

[22] X. Ge, H. Vijayakumar, and T. Jaeger. Sprobes: Enforcing kernel code integrity on the trustzone architecture. 2014.

[23] G. Gogniat, T. Wolf, W. Burleson, J.-P. Diguet, L. Bossuet, and R. Vaslin. Reconfigurable hardware for high-security/high-performance embedded systems: the safes perspective. Very Large Scale Integration (VLSI) Systems, IEEE Transactions on, 16(2):144-155, 2008.

[24] R. Guanciale, H. Nemati, C. Baumann, and M. Dam. Cache Storage Channels: Alias-Driven Attacks and Verified Countermeasures. In The 37th IEEE Symposium on Security and Privacy (SEP), SAN JOSE, CA, May 23-25 2016. IEEE.

[25] J. Halderman, S. Schoen, N. Heninger, W. Clarkson, W. Paul, J. Calandrino, A. Feldman, J. Appelbaum, and E. Felten. Lest We Remember: Cold Boot Attacks on Encryption Keys. In 17th USENIX Security Symposium, pages 45-60, 2008.

[26] O. S. Hofmann, S. Kim, A. M. Dunn, M. Z. Lee, and E. Witchel. Inktag: Secure applications on an untrusted operating system. In ASPLOS'13, 2013, pages 265-278, New York, NY, USA, 2013. ACM.

[27] D. Hulton. Cardbus bus-mastering: 0wning the laptop. In Annual ShmooCon Convention, 2006.

[28] D. E. King. Dlib-ml: A machine learning toolkit. J. Mach. Learn. Res., 10:1755-1758, Dec. 2009.

[29] A. Kopytov. SysBench: A System Performance Benchmark, 2004. https://github.com/akopytov/sysbench.

[30] Y. LeCun, C. Cortes, and C. J. Burges. The mnist database of handwritten digits, 1998. http://yann.lecun.com/exdb/mnist/.

[31] M. Lipp, D. Gruss, R. Spreitzer, C. Maurice, and S. Mangard. Armageddon: Cache attacks on mobile devices. In 25th USENIX Security Symposium (USENIX Security 16), pages 549-564, Austin, TX, Aug. 2016. USENIX Association.

[32] D. Liu and L. P. Cox. Veriui: Attested login for 
mobile devices. In Proceedings of the 15th Workshop on Mobile Computing Systems and Applications, page 7. ACM, 2014.

[33] J. M. McCune, Y. Li, N. Qu, Z. Zhou, A. Datta, V. Gligor, and A. Perrig. Trustvisor: Efficient tcb reduction and attestation. In 2010 IEEE Symposium on Security and Privacy, pages 143-158, May 2010.

[34] J. M. McCune, B. J. Parno, A. Perrig, M. K. Reiter, and H. Isozaki. Flicker: An execution infrastructure for tcb minimization. SIGOPS Oper. Syst. Rev., 42(4):315-328, Apr. 2008.

[35] F. McKeen, I. Alexandrovich, A. Berenzon, C. V. Rozas, H. Shafi, V. Shanbhogue, and U. R. Savagaonkar. Innovative instructions and software model for isolated execution. In Proceedings of the 2Nd International Workshop on Hardware and Architectural Support for Security and Privacy, HASP '13, pages 10:1-10:1, New York, NY, USA, 2013. ACM.

[36] L. McVoy and C. Staelin. Lmbench: Portable tools for performance analysis. In Proceedings of the 1996 Annual Conference on USENIX Annual Technical Conference, ATEC '96, pages 23-23, Berkeley, CA, USA, 1996. USENIX Association.

[37] T. Müller, M. Spreitzenbarth, and F. Freiling. FROST: Forensic recovery of scrambled telephones. In 11th International Conference on Applied Cryptography and Network Security, pages 373-388, 2013.

[38] Samsung Electronics. The KNOX Workspace Technical Details. https://www.samsungknox.com/en/ products/knox-workspace/technical.

[39] N. Santos, H. Raj, S. Saroiu, and A. Wolman. Using arm trustzone to build a trusted language runtime for mobile applications. In $A S P L O S^{\prime} 14,2014$, pages 67-80, New York, NY, USA, 2014. ACM.

[40] P. Schwabe. Eliminating Timing Side-Channels. A Tutorial, 2015.

[41] V. D. Silva, D. Kroening, and G. Weissenbacher. A survey of automated techniques for formal software verification. Computer-Aided Design of Integrated Circuits and Systems, IEEE Transactions on, 27(7):1165-1178, 2008.

[42] H. Sun, K. Sun, Y. Wang, and J. Jing. Trustotp: Transforming smartphones into secure one-time password tokens. In $A C M C C S^{\prime} 15$, 2015, pages 976-988. ACM, 2015.

[43] R. Ta-Min, L. Litty, and D. Lie. Splitting interfaces: Making trust between applications and operating systems configurable. In OSDI'06, 2006, pages 279-292. USENIX Association, 2006.

[44] Wolfgang Denk. Das U-Boot - the Universal Boot Loader. http://www.denx.de/wiki/U-Boot/WebHome.

[45] Y. Xu, W. Cui, and M. Peinado. Controlled-channel attacks: Deterministic side channels for untrusted operating systems. In 2015 IEEE Symposium on Security and Privacy, pages 640-656, May 2015.

[46] J. Yang and K. G. Shin. Using hypervisor to provide data secrecy for user applications on a per-page basis. In Proceedings of the Fourth ACM

SIGPLAN/SIGOPS International Conference on Virtual Execution Environments, VEE '08, pages 71-80, New York, NY, USA, 2008. ACM.
[47] Y. Yarom and K. Falkner. FLUSH+RELOAD: A High Resolution, Low Noise, L3 Cache Side-Channel Attack. In USENIX Security'14, 2014, pages 719-732, San Diego, CA, Aug. 2014. USENIX Association.

[48] N. Zhang, K. Sun, W. Lou, and T. Hou. Case: Cache-assisted secure execution on arm processors. In The 37th IEEE Symposium on Security and Privacy (SEPP), SAN JOSE, CA, May 23-25 2016. IEEE.

[49] Y. Zhang, A. Juels, M. K. Reiter, and T. Ristenpart. Cross-tenant side-channel attacks in paas clouds. In $A C M$ CCS '14, 2014, New York, NY, USA, 2014. ACM.

\section{APPENDIX \\ A. CODE SNIPPET OF EXCEPTION FOR- WARDING}

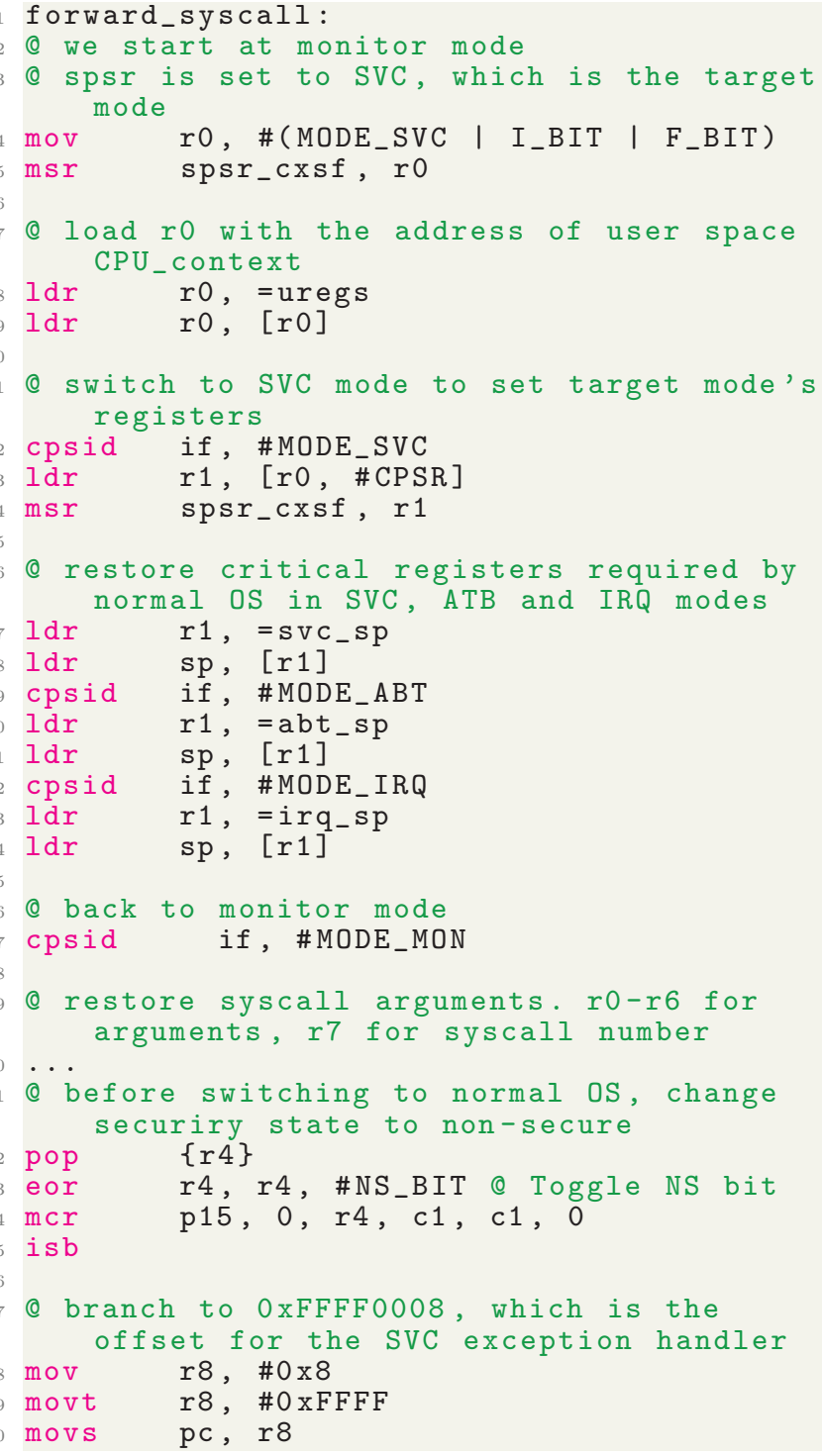

Listing 1: Code snippet that forwards SVC exception to the normal OS. 


\section{B. RAW HTTP PERFORMANCE MEA- SUREMENTS}

\begin{tabular}{|c|c|c|c|c|}
\hline \multirow{2}{*}{ File size } & \multicolumn{2}{|c|}{ Throughput (Requests/second) } & \multicolumn{2}{c|}{$95 \%$ Percentile (ms) } \\
\cline { 2 - 5 } & Linux & TrustShadow & Linux & TrustShadow \\
\hline $1 \mathrm{~KB}$ & 652.50 & 600.46 & 17 & 17 \\
\hline $2 \mathrm{~KB}$ & 622.83 & 567.96 & 18 & 18 \\
\hline $4 \mathrm{~KB}$ & 621.72 & 569.37 & 16 & 18 \\
\hline $8 \mathrm{~KB}$ & 603.75 & 548.74 & 17 & 19 \\
\hline $16 \mathrm{~KB}$ & 531.15 & 488.64 & 19 & 21 \\
\hline $32 \mathrm{~KB}$ & 433.68 & 408.25 & 24 & 25 \\
\hline $64 \mathrm{~KB}$ & 316.64 & 299.57 & 33 & 36 \\
\hline $128 \mathrm{~KB}$ & 220.73 & 213.25 & 48 & 49 \\
\hline $256 \mathrm{~KB}$ & 133.26 & 130.58 & 84 & 85 \\
\hline $512 \mathrm{~KB}$ & 75.85 & 75.69 & 158 & 158 \\
\hline $1024 \mathrm{~KB}$ & 42.68 & 42.64 & 307 & 309 \\
\hline
\end{tabular}

